\title{
Pulmonary eosinophilia associated with cutaneous larva migrans
}

\author{
RJA BUTLAND, IH COULSON \\ From the Department of Medicine I, St George's Hospital Medical School, and the Skin Department, St \\ George's Hospital, London
}

Transient pulmonary eosinophilia (synonyms Loeffler's syndrome, pulmonary infiltration with eosinophilia) was observed in a patient with cutaneous larva migrans (synonyms creeping eruption, plumber's itch), a rare association only previously reported from the United States. ${ }^{12}$

\section{Case report}

A 58 year old non-smoking caucasian woman noticed an itchy rash on her buttocks on 29 October 1983, two days before returning to London from her holiday in Barbados. Her travelling companions were unaffected. On her return similar lesions developed on her legs and abdomen. On 15 November she noticed a cough, which was dry until 25 November, when she produced a little white sputum. There was no wheeze, breathlessness, or haemoptysis. She was anorexic and lost $4.4 \mathrm{~kg}$ in weight. Bowel function was normal. Her medication consisted of atenolol $100 \mathrm{mg}$ and chlorthalidone $25 \mathrm{mg}$ daily for hypertension. There was no history of allergic conditions such as rhinitis, asthma, or eczema.

On examination on 8 November she had at least 20 slightly raised, erythematous, serpiginous burrows typical of cutaneous larva migrans on her abdomen, buttocks, and legs (fig). Treatment with antipruritic drugs did not help

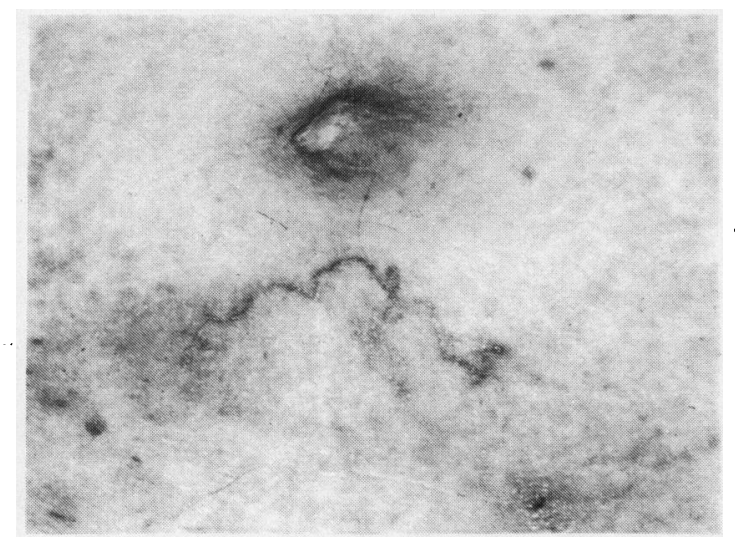

Characteristic serpiginous tract of cutaneous larva migrans on the patient's abdomen just below the umbilicus.

Address for reprint requests: Dr RJA Butland, Skin Department, St George's Hospital, London SW17 0QT.

Accepted 24 September 1984 but topical thiobendazole on 11 November led to a prompt improvement in the skin lesions. Ova, cysts, and parasites were not present in the stool. On 18 November, three weeks after the rash had appeared, the white cell count was $10.4 \times 10^{-9} / 1\left(4.6 \times 10^{-9} / 1\right.$ neutrophils and $3.0 \times 10^{-9} / 1$ eosinophils) and a chest radiograph showed ill defined patchy shadowing in the left upper and middle zones, consistent with an inflammatory process. The right lung was clear. On 25 November the white cell count was $14.3 \times 10^{-9} / 1\left(10.5 \times 10^{-9} / 1\right.$ neutrophils and $1.1 \times 10^{-9} / 1$ eosinophils/l). On 9 December the chest radiograph showed only minimal consolidation persisting behind the left first rib. The left mid zone consolidation had cleared. At this stage she was still coughing but the skin lesions were healing. By January 1984 the rash had resolved, the chest radiograph was clear, and the serum IgE concentration was normal and there was no blood eosinophilia.

\section{Discussion}

Cutaneous larva migrans is usually caused by the larvae of the nematode Ancylostoma braziliense, the dog or cat hookworm. Dogs and cats contaminate the ground through their faeces, which contain ova. These ova progress to active filariform larvae, which penetrate human skin. The larvae appear unable in most cases to penetrate the dermis of human skin and they wander aimlessly in the lower portion of the epidermis for two to 50 weeks before they die.

Simple pulmonary eosinophilia (Loeffler's syndrome) has been associated with the following parasites: Ascaris, Trichuris, Trichiura, Strongyloides, Taenia saginata, Fasciola hepatica, Entamoeba histolytica, Trichinella, Brucella.' Wright and Gold's prospective study of cutaneous larva migrans in 1946 found 26 cases of pulmonary infiltration among 76 cases of creeping eruption, with almost complete absence of clinical signs or symptoms of systemic disease. Only $12 \%$ of patients experienced a cough. There have only been two subsequent case reports, indicating the rarity of symptomatic pulmonary infiltration.

The pathogenesis of the pulmonary infiltrates remains unclear. Muhleisen found $A$ braziliense larvae in the sputum of one patient with larva migrans for 24 days but, although he developed asthma and systemic eosinophilia, there were no pulmonary infiltrates. ${ }^{3}$ On the other hand, Wright and Gold examined 381 sputum specimens from 76 patients with larva migrans (26 with pulmonary eosinophilia) without identifying nematodal larvae. Our patient was unable to produce sputum for analysis. Thus there is scant evidence that pulmonary infiltration is 
associated with the presence of larvae in the lungs (as has been suggested with Ascaris lumbricoides). Possibly the hookworm in the skin led to a generalised sensitisation and reaction with soluble antigen in the lung produced the eosinophilic pulmonary infiltration. The resolution of the pulmonary shadows after the skin was treated with thiobendazole is consistent with this hypothesis.

Cutaneous larva migrans is a fairly common eruption in tropical and subtropical areas. With increasing international travel it may be seen more frequently in Britain in the future, and physicians should be aware of the pulmonary complications.

We are grateful to Professor KB Saunders and to Dr KV Sanderson for permission to report the case and for read- ing the manuscript, and to Mrs R Perry for typing the manuscript.

\section{References}

1 Wright DO, Gold EM. Loeffler's syndrome associated with creeping eruption (cutaneous helminthiasis). Arch Intern Med 1946; 78:303-12.

2 Guill MA, Odon RB. Larva migrans complicated by Loeffler's syndrome. Arch Dermatol 1978;114:1525-6.

3 Muhleisen JP. Demonstration of pulmonary migration of the causative organism of creeping eruption. Ann Intern Med 1953:38: 595-600.

\section{Book notices}

The Lung: Radiologic-pathologic Correlations. 2nd ed. E Robert Heitzman. (Pp 546; £56.) C V Mosby Company. 1984.

Because clinicians, radiologists, and pathologists diagnose pulmonary lesions in different ways, they frequently have difficulty in communicating with each other. This book, now in its second edition, cuts across the specialties and effectively bridges the gaps between them. The opening chapters deal with techniques for the preparation of lung specimens, embryology, anatomy, developmental anomalies, and radiological pattern recognition. These are followed by sections on circulatory, inflammatory, and neoplastic disorders. The last chapter covers pleural disease. The text throughout is lucid, the illustrations are of high quality, and each topic is followed by a comprehensive list of references. Dr Hertzman states rather apologetically that it is not his intention to produce an all embracing atlas of correlated pulmonary pathology. Coincidentally he has succeeded in doing so, although he goes much further than the standard bench books. His presentation is factual, but at the same time thought provoking and open ended, avoiding the dead hand of rigid classification. For this reason The Lung will not suit candidates preparing for multiple choice examinations, nor those unfortunates possessed of slot machine minds. It is, however, recommended reading for anyone seeking a more flexible approach to the diagnosis of chest disease.-CWF
Immunopathologie Broncho-pulmonaire. 2nd ed. C Molina. (Pp 319; price not known.) Masson. 1984.

This French language text provides a comprehensive review of manageable length of the immunological aspects of a wide variety of lung disorders. After a preliminary section which deals in general with hypersensitivity responses, host defence mechanisms, and methods of investigating these the remainder is subdivided into two sections. The first deals with immunological disorders where the brunt of the disease is borne by the parenchyma and the second section concentrates on asthma, with a final chapter on pleural disorders. The style is difficult to assess in a foreign text but the pleasures of encountering "Le poumon du Neffa" in the allergic alveolitis section makes the effort worthwhile. The section on allergic alveolitis in particular is extremely comprehensive but the chapters on pulmonary vasculitis, fibrosing alveolitis, and connective tissue disorders are disappointingly slim. The illustrations are, on the whole, adequate, with some splendid photomicrographs, but the presentation of radiographs in the positive form is unhelpful. There are several equally good sources of pulmonary immunopathology in the English language and this text will therefore have limited appeal to those with a modest command of French. For those with the time and the talent to struggle through it, however, it provides an interesting insight into the cross-Channel approach to an important aspect of pulmonary disease.RMAB 